\title{
Variational Iteration Method for Singular Perturbation Initial Value Problems with Delays
}

\author{
Yongxiang Zhao, ${ }^{1,2}$ Aiguo Xiao, ${ }^{3} \mathrm{Li} \mathrm{Li}^{2}$ and Chengjian Zhang' \\ ${ }^{1}$ School of Mathematics and Statistics, Huazhong University of Science \& Technology, Wuhan 430074, China \\ ${ }^{2}$ School of Mathematics and Statistics, Chongqing Three Gorges University, Wanzhou 404000, China \\ ${ }^{3}$ School of Mathematics and Computational Science, Hunan Key Laboratory for Computation and Simulation in Science and \\ Engineering, Xiangtan University, Xiangtan, Hunan 411105, China
}

Correspondence should be addressed to Chengjian Zhang; cjzhang@mail.hust.edu.cn

Received 7 October 2013; Revised 21 December 2013; Accepted 21 December 2013; Published 5 March 2014

Academic Editor: Sotiris. K. Ntouyas

Copyright (c) 2014 Yongxiang Zhao et al. This is an open access article distributed under the Creative Commons Attribution License, which permits unrestricted use, distribution, and reproduction in any medium, provided the original work is properly cited.

The variational iteration method (VIM) is applied to solve singular perturbation initial value problems with delays (SPIVPDs). Some convergence results of VIM for solving SPIVPDs are given. The obtained sequence of iterates is based on the use of general Lagrange multipliers; the multipliers in the functionals can be identified by the variational theory. Moreover, the numerical examples show the efficiency of the method.

\section{Introduction}

Singular perturbation initial value problems with delays play an important role in the research of various applied sciences, such as control theory, population dynamics, medical science, environment science, biology, and economics $[1,2]$. These problems are characterized by a small parameter $\epsilon$ multiplying the highest derivatives, and the state variables depend not only on the value of the time, but also on the value prior to the time. Because the classical Lipschitz constant and onesided Lipschitz constant are generally of size $\mathcal{O}\left(\epsilon^{-1}\right)(0<$ $\epsilon \ll 1)$, the classical convergence theory, B-convergence theory, and D-convergence theory cannot be directly applied to SPIVPDs.

Starting from pioneering ideas going back to InokutiSekine-Mura method [3], the variational iteration method was first proposed in later 1990s by $\mathrm{He}$ [4-6]. By recent years, this method has been extensively applied to various ODEs, integral equations, delay differential equations and fractional differential equations, two-point boundary value problems, oscillations, and stiff ODEs, notably, He [7, 8], Wazwaz $[9,10]$, Draganescu et al. [11, 12], Saadatmandi and Dehghan [13], Salkuyeh [14], Lu [15], Xu [16], Rafei et al. [17], Darvishi et al. [18], Tatari and Dehghan [19], Mamode [20], Saadati and Dehghan [21], Yu [22], Marinca et al. [23], Yang and Dumitru
[24], and $\mathrm{Wu}$ [25] to mention only a few. Recently, Zhao and Xiao [26] have applied this method for solving singular perturbation initial value problems. For more comprehensive survey on this method and its applications, the reader is referred to the review articles [26-28] and the references therein.

In this paper, we apply the VIM to SPIVPDs to obtain the analytical or approximate analytical solutions. The convergence results of VIM for solving SPIVPDs are obtained. Some illustrative examples confirm the theoretical results.

In the remaining parts of the text, we denote $x(t)=$ $x(t, \epsilon), y(t)=y(t, \epsilon)$ for simplicity; here, $\epsilon$ is the singular perturbation parameter. The vectors $\left(a_{1}, a_{2}, \ldots, a_{n}\right)^{T}=a \leq$ $b=\left(b_{1}, b_{2}, \ldots, b_{n}\right)^{T}$ mean that each component $a_{i} \leq b_{i}(i=$ $1,2, \ldots, n) .\|\cdot\|$ denotes the standard Euclidean norm of a vector.

\section{Convergence Analysis}

2.1. Case 1. Consider the following singular perturbation initial value problem with delays:

$$
\begin{array}{r}
x^{\prime}(t)=f(t, x(t), x(t-\tau), y(t), y(t-\tau)), \\
0 \leq t \leq T,
\end{array}
$$




$$
\begin{gathered}
\epsilon y^{\prime}(t)=g(t, x(t), x(t-\tau), y(t), x(t-\tau)), \\
0<\epsilon \ll 1, \\
x(t)=\varphi(t), \quad y(t)=\psi(t), \quad t \leq 0,
\end{gathered}
$$

where $x \in R^{n_{1}}$ and $y \in R^{n_{2}}$ are the state variables and $\epsilon$ is the singular perturbation parameter. $f:[-\tau, T] \times R^{n_{1}} \times R^{n_{1}} \times$ $R^{n_{2}} \times R^{n_{2}} \rightarrow R^{n_{1}}, g:[-\tau, T] \times R^{n_{1}} \times R^{n_{1}} \times R^{n_{2}} \times R^{n_{2}} \rightarrow R^{n_{2}}$ are given continuous mappings which satisfy the following Lipschitz conditions:

$$
\begin{gathered}
\left\|f\left(t, x_{1}, u_{1}, y_{1}, v_{1}\right)-f\left(t, x_{2}, u_{2}, y_{2}, v_{2}\right)\right\| \\
\leq l_{1}(t)\left\|x_{1}-x_{2}\right\|+l_{2}(t)\left\|u_{1}-u_{2}\right\| \\
\quad+l_{3}(t)\left\|y_{1}-y_{2}\right\|+l_{4}(t)\left\|v_{1}-v_{2}\right\|, \\
\left\|g\left(t, x_{1}, u_{1}, y_{1}, v_{1}\right)-g\left(t, x_{2}, u_{2}, y_{2}, v_{2}\right)\right\| \\
\leq k_{1}(t)\left\|x_{1}-x_{2}\right\|+k_{2}(t)\left\|u_{1}-u_{2}\right\| \\
\quad+k_{3}(t)\left\|y_{1}-y_{2}\right\|+k_{4}(t)\left\|v_{1}-v_{2}\right\|,
\end{gathered}
$$

where $l_{i}(t), k_{i}(t)(i=1, \ldots, 4)$ are continuous bounded functions.

According to VIM, we can construct the correction functionals as follows:

$$
\begin{aligned}
& x_{n+1}(t)=x_{n}(t) \\
& +\int_{0}^{t} \lambda_{1}(s, t) \\
& \times\left(x_{n}^{\prime}(s)-\tilde{f}\left(s, x_{n}(s), x_{n}(s-\tau), y_{n}(s),\right.\right. \\
& \left.\left.y_{n}(s-\tau)\right)\right) d s \text {, } \\
& y_{n+1}(t)=y_{n}(t) \\
& +\int_{0}^{t} \lambda_{2}(s, t) \\
& \times\left(y_{n}^{\prime}(s)-\frac{1}{\epsilon} \tilde{g}\left(s, x_{n}(s), x_{n}(s-\tau),\right.\right. \\
& \left.\left.y_{n}(s), y_{n}(s-\tau)\right)\right) d s,
\end{aligned}
$$

where $\lambda_{1}(s, t), \lambda_{2}(s, t)$ are general Lagrange multipliers, which can be defined optimally via variational theory, and $\tilde{f}, \tilde{g}$ denote the restrictive variation; that is, $\delta \tilde{f}=\delta \widetilde{g}=\mathbf{0}$. Thus, we have

$$
\begin{aligned}
& \delta x_{n+1}(t)=\delta x_{n}(t)+\int_{0}^{t} \lambda_{1}(s, t) \delta x_{n}^{\prime}(s) d s, \\
& \delta y_{n+1}(t)=\delta y_{n}(t)+\int_{0}^{t} \lambda_{2}(s, t) \delta y_{n}^{\prime}(s) d s,
\end{aligned}
$$

and the stationary conditions are obtained as

$$
\begin{array}{cl}
1+\left.\lambda_{1}(s, t)\right|_{s=t}=0, & \frac{\partial \lambda_{1}(s, t)}{\partial s}=0, \\
1+\left.\lambda_{2}(s, t)\right|_{s=t}=0, & \frac{\partial \lambda_{2}(s, t)}{\partial s}=0 .
\end{array}
$$

Moreover, the general Lagrange multiplier can be readily identified by

$$
\lambda_{1}(s, t)=\lambda_{2}(s, t)=-1 .
$$

Therefore, the variational iteration formulas can be written as

$$
\begin{aligned}
& x_{n+1}(t)=x_{n}(t)-\int_{0}^{t}\left(x_{n}^{\prime}(s)\right. \\
& -f\left(s, x_{n}(s), x_{n}(s-\tau),\right. \\
& \left.\left.y_{n}(s), y_{n}(s-\tau)\right)\right) d s, \\
& y_{n+1}(t)=y_{n}(t) \\
& -\int_{0}^{t}\left(y_{n}^{\prime}(s)\right. \\
& -\frac{1}{\epsilon} g\left(s, x_{n}(s),\right. \\
& \left.\left.x_{n}(s-\tau), y_{n}(s), y_{n}(s-\tau)\right)\right) d s .
\end{aligned}
$$

Now, we show that the iterative sequences $\left\{x_{n}(t)\right\}_{n=1}^{\infty}$, $\left\{y_{n}(t)\right\}_{n=1}^{\infty}$ defined by (7a) and (7b) with $x_{0}(t)=\varphi(t), y_{0}(t)=$ $\psi(t)$ converge to the solution of (1).

Theorem 1. Let $x(t), x_{i}(t) \in\left(C^{1}[0, T]\right)^{n_{1}}, y(t), y_{i}(t) \in\left(C^{1}[0\right.$, $T])^{n_{2}}, i=0,1, \ldots$. The sequences defined by (7a) and (7b) with $x_{0}(t)=\varphi(t), y_{0}(t)=\psi(t)$ converge to the solution of $(1)$.

Proof. Obviously from system (1), we have

$$
\begin{aligned}
x(t)= & x(t) \\
& -\int_{0}^{t}\left(x^{\prime}(s)\right. \\
& -f(s, x(s), x(s-\tau), y(s), y(s-\tau))) d s, \\
y(t)= & y(t) \quad-\int_{0}^{t}\left(y^{\prime}(s)\right. \\
& \left.\quad-\frac{1}{\epsilon} g(s, x(s), x(s-\tau), y(s), y(s-\tau))\right) d s .
\end{aligned}
$$

Introduce $E_{i} x(t)=x_{i}(t)-x(t), E_{i} y(t)=y_{i}(t)-y(t), E_{i} x(t-$ $\tau)=x_{i}(t-\tau)-x(t-\tau), E_{i} y(t-\tau)=y_{i}(t-\tau)-y(t-\tau), i=$ $0,1, \ldots$, where $E_{j} x(0)=E_{j} y(0)=0, j=0,1, \ldots$. Now from (7a), (7b)-(8a), and (8b) we obtain

$$
\begin{array}{r}
E_{n+1} x(t)=E_{n} x(t) \\
-\int_{0}^{t}\left(E_{n} x^{\prime}(s)-\left(f \left(s, x_{n}(s), x_{n}(s-\tau),\right.\right.\right. \\
\left.y_{n}(s), y_{n}(s-\tau)\right) \\
-f(s, x(s), x(s-\tau), \\
y(s), y(s-\tau)))) d s,
\end{array}
$$


$E_{n+1} y(t)=E_{n} y(t)$

$$
\begin{gathered}
-\int_{0}^{t}\left(E_{n} y^{\prime}(s)-\frac{1}{\epsilon}\right. \\
\times\left(g \left(s, x_{n}(s), x_{n}(s-\tau),\right.\right. \\
\left.y_{n}(s), y_{n}(s-\tau)\right) \\
-g(s, x(s), x(s-\tau), y(s), \\
y(s-\tau)))) d s .
\end{gathered}
$$

(9)

Moreover, we can derive

$$
\begin{gathered}
E_{n+1} x(t)=\int_{0}^{t}\left(f\left(s, x_{n}(s), x_{n}(s-\tau), y_{n}(s), y_{n}(s-\tau)\right)\right. \\
-f(s, x(s), x(s-\tau), y(s), \\
y(s-\tau))) d s, \\
E_{n+1} y(t)=\frac{1}{\epsilon} \int_{0}^{t}\left(g\left(s, x_{n}(s), x_{n}(s-\tau), y_{n}(s), y_{n}(s-\tau)\right)\right. \\
-g(s, x(s), x(s-\tau), y(s), \\
y(s-\tau))) d s .
\end{gathered}
$$

Now, the integration interval is split into two parts

$$
\begin{aligned}
& E_{n+1} x(t) \\
& =\int_{0}^{\tau}\left(f\left(s, x_{n}(s), \varphi(s-\tau), y_{n}(s), \psi(s-\tau)\right)\right. \\
& -f(s, x(s), \varphi(s-\tau) \\
& y(s), \psi(s-\tau))) d s \\
& +\int_{\tau}^{t}\left(f\left(s, x_{n}(s), x_{n}(s-\tau), y_{n}(s), y_{n}(s-\tau)\right)\right. \\
& -f(s, x(s), x(s-\tau), \\
& y(s), y(s-\tau))) d s
\end{aligned}
$$

$$
\begin{aligned}
& E_{n+1} y(t) \\
& =\frac{1}{\epsilon}\left(\int _ { 0 } ^ { \tau } \left(g\left(s, x_{n}(s), \varphi(s-\tau), y_{n}(s), \psi(s-\tau)\right)\right.\right. \\
& \quad-g(s, x(s), \varphi(s-\tau), y(s), \psi(s-\tau))) d s \\
& +\int_{\tau}^{t}\left(g\left(s, x_{n}(s), x_{n}(s-\tau), y_{n}(s), y_{n}(s-\tau)\right)\right. \\
& \quad-g(s, x(s), x(s-\tau), y(s), y(s-\tau))) d s) .
\end{aligned}
$$

From the Lipschitz conditions (2a) and (2b), we have

$$
\begin{aligned}
\left(\begin{array}{ll}
\left\|E_{n+1} x(t)\right\| \\
\left\|E_{n+1} y(t)\right\|
\end{array}\right) \leq & \left(\begin{array}{ll}
l_{1} & l_{3} \\
\frac{k_{1}}{\epsilon} & \frac{k_{3}}{\epsilon}
\end{array}\right)\left(\begin{array}{l}
\int_{0}^{t}\left\|E_{n} x(s)\right\| d s \\
\int_{0}^{t}\left\|E_{n} y(s)\right\| d s
\end{array}\right) \\
& +\left(\begin{array}{ll}
l_{2} & l_{4} \\
\frac{k_{2}}{\epsilon} & \frac{k_{4}}{\epsilon}
\end{array}\right)\left(\begin{array}{l}
\int_{0}^{t}\left\|E_{n} x(s-\tau)\right\| d s \\
\int_{0}^{t}\left\|E_{n} y(s-\tau)\right\| d s
\end{array}\right),
\end{aligned}
$$

where $l_{i}=\max _{0 \leq s \leq T} l_{i}(s), k_{i}=\max _{0 \leq s \leq T} k_{i}(s), i=1, \ldots, 4$. Therefore,

$$
\begin{aligned}
& \left(\begin{array}{l}
\left\|E_{1} x(t)\right\| \\
\left\|E_{1} y(t)\right\|
\end{array}\right) \leq\left(\begin{array}{ll}
l_{1} & l_{3} \\
\frac{k_{1}}{\epsilon} & \frac{k_{3}}{\epsilon}
\end{array}\right)\left(\begin{array}{l}
\int_{0}^{t}\left\|E_{0} x(s)\right\| d s \\
\int_{0}^{t}\left\|E_{0} y(s)\right\| d s
\end{array}\right) \\
& +\left(\begin{array}{ll}
l_{2} & l_{4} \\
\frac{k_{2}}{\epsilon} & \frac{k_{4}}{\epsilon}
\end{array}\right)\left(\begin{array}{l}
\int_{0}^{t}\left\|E_{0} x(s-\tau)\right\| d s \\
\int_{0}^{t}\left\|E_{0} y(s-\tau)\right\| d s
\end{array}\right) \\
& \leq\left(\begin{array}{cc}
l_{1}+l_{2} & l_{3}+l_{4} \\
\frac{k_{1}+k_{2}}{\epsilon} & \frac{k_{3}+k_{4}}{\epsilon}
\end{array}\right)\left(\begin{array}{c}
\max _{-\tau \leq s \leq T}\left\|E_{0} x(s)\right\| t \\
\max _{-\tau \leq s \leq T}\left\|E_{0} y(s)\right\| t
\end{array}\right) .
\end{aligned}
$$

Moreover, we can derive

$$
\begin{aligned}
& \left(\begin{array}{l}
\left\|E_{n} x(t)\right\| \\
\left\|E_{n} y(t)\right\|
\end{array}\right) \leq \frac{T^{n}}{n !}\left(\begin{array}{cc}
l_{1}+l_{2} & l_{3}+l_{4} \\
\frac{k_{1}+k_{2}}{\epsilon} & \frac{k_{3}+k_{4}}{\epsilon}
\end{array}\right)^{n} \\
& \times\left(\begin{array}{c}
\max _{-\tau \leq s \leq T}\left\|E_{0} x(s)\right\| \\
\max _{-\tau \leq s \leq T}\left\|E_{0} y(s)\right\|
\end{array}\right) \\
& \leq \frac{(T / \epsilon)^{n}}{n !}\left(\begin{array}{ll}
\mathcal{O}(\epsilon) & \mathcal{O}(\epsilon) \\
\mathcal{O}(1) & \mathcal{O}(1)
\end{array}\right)\left(\begin{array}{c}
\max _{-\tau \leq s \leq T}\left\|E_{0} x(s)\right\| \\
\max _{-\tau \leq s \leq T}\left\|E_{0} y(s)\right\|
\end{array}\right)
\end{aligned}
$$

Noting that $\epsilon, T, \max _{-\tau \leq s \leq T}\left\|E_{0} x(s)\right\|, \max _{-\tau \leq s \leq T}\left\|E_{0} y(s)\right\|, l_{i}$, $k_{i}, i=1, \ldots, 4$ are constants. By using Stirling's formula, we have

$$
\begin{aligned}
\left(\begin{array}{l}
\left\|E_{n} x(t)\right\| \\
\left\|E_{n} y(t)\right\|
\end{array}\right) \leq & \frac{((T e / \epsilon) / n)^{n}}{\sqrt{2 \pi n}(1+\mathcal{O}(1 / n))} \\
& \times\left(\begin{array}{cc}
\mathcal{O}(\epsilon) & \mathcal{O}(\epsilon) \\
\mathcal{O}(1) & \mathcal{O}(1)
\end{array}\right)\left(\begin{array}{c}
\max _{-\tau \leq s \leq T}\left\|E_{0} x(s)\right\| \\
\max _{-\tau \leq s \leq T}\left\|E_{0} y(s)\right\|
\end{array}\right)
\end{aligned}
$$

thus, $\left(\left\|E_{n} x(t)\right\|,\left\|E_{n} y(t)\right\|\right)^{T} \rightarrow \mathbf{0}$ as $n \rightarrow \infty$. 
2.2. Case 2. Consider the special case of (1):

$$
\begin{array}{r}
x^{\prime}(t)=A x(t)+F(t, x(t), x(t-\tau), y(t), y(t-\tau)), \\
0 \leq t \leq T, \\
\epsilon y^{\prime}(t)=B y(t)+G(t, x(t), x(t-\tau), y(t), y(t-\tau)), \\
0<\epsilon \ll 1, \\
x(t)=\varphi(t), \quad y(t)=\psi(t), \quad t \leq 0,
\end{array}
$$

where $F:[-\tau, T] \times R^{n_{1}} \times R^{n_{1}} \times R^{n_{2}} \times R^{n_{2}} \rightarrow R^{n_{1}}, G:$ $[-\tau, T] \times R^{n_{1}} \times R^{n_{1}} \times R^{n_{2}} \times R^{n_{2}} \rightarrow R^{n_{2}}$ are given continuous mappings which satisfy the Lipschitz conditions (2a) and (2b); the matrices $A=\left(a_{i j}\right) \in R^{n_{1} \times n_{1}}, B=\left(b_{i j}\right) \in R^{n_{2} \times n_{2}}$ can be decomposed into $A=A_{0}+A_{1}, B=B_{0}+B_{1}$, respectively, where $A_{0}=\operatorname{diag}\left(a_{11}, a_{22}, \ldots, a_{n_{1} n_{1}}\right)$ and $B_{0}=$ $\operatorname{diag}\left(b_{11}, b_{22}, \ldots, b_{n_{2} n_{2}}\right)$ :

$$
\begin{gathered}
\left\|F\left(t, x_{1}, u_{1}, y_{1}, v_{1}\right)-F\left(t, x_{2}, u_{2}, y_{2}, v_{2}\right)\right\| \\
\leq p_{1}(t)\left\|x_{1}-x_{2}\right\|+p_{2}(t)\left\|u_{1}-u_{2}\right\| \\
\quad+p_{3}(t)\left\|y_{1}-y_{2}\right\|+p_{4}(t)\left\|v_{1}-v_{2}\right\|, \\
\left\|G\left(t, x_{1}, u_{1}, y_{1}, v_{1}\right)-G\left(t, x_{2}, u_{2}, y_{2}, v_{2}\right)\right\| \\
\leq q_{1}(t)\left\|x_{1}-x_{2}\right\|+q_{2}(t)\left\|u_{1}-u_{2}\right\| \\
\quad+q_{3}(t)\left\|y_{1}-y_{2}\right\|+q_{4}(t)\left\|v_{1}-v_{2}\right\|,
\end{gathered}
$$

where $p_{i}(t), q_{i}(t)(i=1, \ldots, 4)$ are continuous bounded functions.

It is easy to show that the right hand sides of (16) also satisfy the Lipschitz conditions. If the right hand sides of (16) are considered as nonlinear terms, then we can also use the correction functionals constructed in Case 1 and get similar results to Theorem 1 . Now, we construct the following correction functionals:

$$
\begin{aligned}
& x_{n+1}(t)= x_{n}(t) \\
&+\int_{0}^{t} \Lambda_{1}(s, t)\left(x_{n}^{\prime}(s)-A_{0} x_{n}(s)-A_{1} \widetilde{x}_{n}(s)\right. \\
&-\widetilde{F}\left(s, x_{n}(s), x_{n}(s-\tau),\right. \\
&\left.\left.y_{n}(s), y_{n}(s-\tau)\right)\right) d s, \\
& y_{n+1}(t)=y_{n}(t) \\
&+\int_{0}^{t} \Lambda_{2}(s, t) \\
& \times\left(y_{n}^{\prime}(s)-\frac{1}{\epsilon}\left(B_{0} y_{n}(s)+B_{1} \widetilde{y}_{n}(s)\right.\right. \\
&+\widetilde{G}\left(s, x_{n}(s), x_{n}(s-\tau),\right. \\
&\left.\left.\left.y_{n}(s), y_{n}(s-\tau)\right)\right)\right) d s,
\end{aligned}
$$

where $\Lambda_{1}(s, t)=\operatorname{diag}\left(\lambda_{11}(s, t), \lambda_{12}(s, t), \ldots, \lambda_{1 n_{1}}(s, t)\right), \Lambda_{2}(s$, $t)=\operatorname{diag}\left(\lambda_{21}(s, t), \lambda_{22}(s, t), \ldots, \lambda_{2 n_{2}}(s, t)\right)$, in which $\lambda_{1 i}(s, t)$, $\lambda_{2 j}(s, t), i=1,2, \ldots, n_{1}, j=1,2, \ldots, n_{2}$ are general Lagrange multipliers and $\widetilde{x}_{n}, \widetilde{y}_{n}, \widetilde{F}, \widetilde{G}$ denote the restrictive variations; that is, $\delta \widetilde{x}_{n}=\delta \widetilde{y}_{n}=\delta \widetilde{F}=\delta \widetilde{G}=\mathbf{0}$. Thus, we have

$$
\begin{aligned}
& \delta x_{n+1}(t)=\delta x_{n}(t)+\int_{0}^{t} \Lambda_{1}(s, t)\left(\delta x_{n}^{\prime}(s)-A_{0} \delta x_{n}(s)\right) d s, \\
& \delta y_{n+1}(t)=\delta y_{n}(t)+\int_{0}^{t} \Lambda_{2}(s, t)\left(\delta y_{n}^{\prime}(s)-\frac{B_{0}}{\epsilon} \delta y_{n}(s)\right) d s,
\end{aligned}
$$

and the stationary conditions are obtained as

$$
\begin{array}{ll}
1+\left.\Lambda_{1}(s, t)\right|_{s=t}=0, & \frac{\partial \Lambda_{1}(s, t)}{\partial s}+A_{0} \Lambda_{1}(s, t)=0, \\
1+\left.\Lambda_{2}(s, t)\right|_{s=t}=0, & \frac{\partial \Lambda_{1}(s, t)}{\partial s}+\frac{B_{0}}{\epsilon} \Lambda_{2}(s, t)=0 .
\end{array}
$$

Moreover, the general Lagrange multipliers can be readily identified by

$$
\begin{aligned}
& \Lambda_{1}(s, t)=-\exp \left(-A_{0}(s-t)\right), \\
& \Lambda_{2}(s, t)=-\exp \left(-\frac{B_{0}}{\epsilon}(s-t)\right) .
\end{aligned}
$$

Therefore, the variational iteration formula can be written as

$$
\begin{aligned}
& x_{n+1}(t)=x_{n}(t) \\
& -\int_{0}^{t} e^{-A_{0}(s-t)}\left(x_{n}^{\prime}(s)-A x_{n}(s)\right. \\
& -F\left(s, x_{n}(s), x_{n}(s-\tau),\right. \\
& \left.\left.y_{n}(s), y_{n}(s-\tau)\right)\right) d s, \\
& =y_{n}(t)-\int_{0}^{t} e^{\left(\left(-B_{0} / \epsilon\right)(s-t)\right)} \\
& \times\left(y_{n}^{\prime}(s)-\frac{1}{\epsilon}\right. \\
& \times\left(B y_{n}(s)\right. \\
& +G\left(s, x_{n}(s), x_{n}(s-\tau), y_{n}(s),\right. \\
& \left.\left.\left.y_{n}(s-\tau)\right)\right)\right) d s .
\end{aligned}
$$

The following theorem shows that the sequences $\left\{x_{n}(t)\right\}_{n=1}^{\infty},\left\{y_{n}(t)\right\}_{n=1}^{\infty}$ defined by (22a) and (22b) with $x_{0}(t)=\varphi(t), y_{0}(t)=\psi(t)$ converge to the solution of (16). 
Theorem 2. Let $x(t), x_{i}(t) \in\left(C^{1}[0, T]\right)^{n 1}, y(t), y_{i}(t) \in\left(C^{1}[0\right.$, $T])^{n 2}, i=0,1, \ldots$. The sequences defined by (22a) and (22b) with $x_{0}(t)=\varphi(t), y_{0}(t)=\psi(t)$ converge to the solutions of (16).

Proof. By a similar process to the proof of Theorem 1, we can easily obtain Obviously from system (16) we have

$$
\begin{gathered}
x(t)=x(t)-\int_{0}^{t}\left(x^{\prime}(s)-A x(t)\right. \\
-F(s, x(s), x(s-\tau), y(s), \\
y(s-\tau))) d s, \\
y(t)=y(t) \quad-\int_{0}^{t}\left(y^{\prime}(s)\right. \\
-\frac{1}{\epsilon}(B y(t) \\
+G(s, x(s), x(s-\tau), y(s), \\
y(s-\tau)))) d s .
\end{gathered}
$$

Introduce $E_{i} x(t)=x_{i}(t)-x(t), E_{i} y(t)=y_{i}(t)-y(t), i=$ $0,1, \ldots$, where $E_{j} x(0)=E_{j} y(0)=0, j=0,1, \ldots$. Now from (22a), (22b)-(23a), and (23b) we obtain

$$
\begin{aligned}
& E_{n+1} x(t) \\
& \begin{array}{l}
=\int_{0}^{t} e^{-A_{0}(s-t)} \\
\quad \times\left(A_{1} E_{n} x(s)\right. \\
\quad+\left(F\left(s, x_{n}(s), x_{n}(s-\tau), y_{n}(s), y_{n}(s-\tau)\right)\right. \\
\quad-F(s, x(s), x(s-\tau), y(s), y(s-\tau)))) d s,
\end{array}
\end{aligned}
$$$$
E_{n+1} y(t)
$$$$
=\int_{0}^{t} e^{\left(\left(-B_{0} / \epsilon\right)(s-t)\right)}
$$$$
\times\left(\frac { 1 } { \epsilon } \left(B_{1} E_{n} y(s)\right.\right.
$$$$
+G\left(s, x_{n}(s), x_{n}(s-\tau), y_{n}(s),\right.
$$$$
\left.y_{n}(s-\tau)\right)
$$$$
-G(s, x(s), x(s-\tau), y(s) \text {, }
$$$$
y(s-\tau)))) d s .
$$

From the Lipschitz conditions (17a) and (17b), we have

$$
\begin{aligned}
\left(\begin{array}{cc}
\left\|E_{n+1} x(t)\right\| \\
\left\|E_{n+1} y(t)\right\|
\end{array}\right) \leq & \left(\begin{array}{cc}
e^{-\left\|A_{0}\right\|(s-t)} & 0 \\
0 & e^{-\left(\left\|B_{0}\right\| / \epsilon\right)(s-t)}
\end{array}\right) \\
& \times\left(\begin{array}{cc}
\left\|A_{1}\right\|+p_{1} & p_{3} \\
\frac{q_{1}}{\epsilon} & \frac{\left\|B_{1}\right\|+q_{3}}{\epsilon}
\end{array}\right) \\
& \times\left(\begin{array}{ll}
\int_{0}^{t}\left\|E_{n} x(s)\right\| d s \\
\int_{0}^{t}\left\|E_{n} y(s)\right\| d s
\end{array}\right) \\
& +\left(\begin{array}{cc}
e^{-\left\|A_{0}\right\|(s-t)} & 0 \\
0 & e^{-\left(\left\|B_{0}\right\| / \epsilon\right)(s-t)}
\end{array}\right) \\
& \times\left(\begin{array}{ll}
p_{2} & p_{4} \\
\frac{q_{2}}{\epsilon} & \frac{q_{4}}{\epsilon}
\end{array}\right) \\
& \times\left(\begin{array}{l}
\int_{0}^{t}\left\|E_{n} x(s-\tau)\right\| d s \\
\int_{0}^{t}\left\|E_{n} y(s-\tau)\right\| d s
\end{array}\right) ;
\end{aligned}
$$

similarly, we can derive

$$
\begin{aligned}
& \left(\begin{array}{l}
\left\|E_{n} x(t)\right\| \\
\left\|E_{n} y(t)\right\|
\end{array}\right) \leq e(\epsilon) \frac{T^{n}}{n !}\left(\begin{array}{cc}
\left\|A_{1}\right\|+p_{1}+p_{2} & p_{3}+p_{4} \\
\frac{q_{1}+q_{2}}{\epsilon} & \frac{\left\|B_{1}\right\|+q_{3}+q_{4}}{\epsilon}
\end{array}\right)^{n} \\
& \times\left(\begin{array}{c}
\max _{-\tau \leq s \leq T}\left\|E_{0} x(s)\right\| \\
\max _{-\tau \leq s \leq T}\left\|E_{0} y(s)\right\|
\end{array}\right) \leq \frac{e(\epsilon)((T e / \epsilon) / n)^{n}}{\sqrt{2 \pi n}(1+\mathcal{O}(1 / n))} \\
& \times\left(\begin{array}{cc}
\mathcal{O}(\epsilon) & \mathcal{O}(\epsilon) \\
\mathcal{O}(1) & \mathcal{O}(1)
\end{array}\right)\left(\begin{array}{c}
\max _{-\tau \leq s \leq T}\left\|E_{0} x(s)\right\| \\
\max _{-\tau \leq s \leq T}\left\|E_{0} y(s)\right\|
\end{array}\right),
\end{aligned}
$$

where $e(\epsilon)=\max _{0 \leq s \leq t, 0 \leq t \leq T}\left(e^{-\left\|A_{0}\right\|(s-t)}, e^{-\left(\left\|B_{0}\right\| / \epsilon\right)(s-t)}\right), p_{i}=$ $\max _{0 \leq s \leq T} p_{i}(s), q_{i}=\max _{0 \leq s \leq T} q_{i}(s), i=1, \ldots, 4$. Noting that $\epsilon, T,\left\|A_{1}\right\|,\left\|B_{1}\right\|, e(\epsilon), p_{i}, q_{i}, i=1, \ldots, 4, \max _{-\tau \leq s \leq T}\left\|E_{0} x(s)\right\|$ and $\max _{-\tau \leq s \leq T}\left\|E_{0} y(s)\right\|$ are constants, we can derive from (26) that $\left(\left\|E_{n} x(t)\right\|,\left\|E_{n} y(t)\right\|\right)^{T} \rightarrow \mathbf{0}$ as $n \rightarrow \infty$.

\section{Numerical Examples}

In this section, some numerical examples are given to show the efficiency of the VIM for solving SPIVPs.

Example 3. Consider SPIVPD (cf. [2]):

$$
x^{\prime}(t)=x(t-1) y(t-1)-1000 x(t)+2 y^{2}(t)+R_{x}(t),
$$

$$
t>0
$$




$$
\begin{gathered}
\epsilon y^{\prime}(t)=x(t-1)-y(t-1)-(1+x(t)) y(t)+R_{y}(t), \\
0<\epsilon \ll 1, \\
x(t)=e^{-0.5 t}+e^{-0.2 t}, \quad y(t)=-e^{-0.5 t}+e^{-0.2 t}, \\
t \leq 0,
\end{gathered}
$$

where

$$
\begin{aligned}
R_{x}(t)= & 999.5 e^{-0.5 t}+999.8 e^{-0.2 t}+e^{-(t-1)} \\
& -e^{-0.4(t-1)}-2 e^{-t}-2 e^{-0.4 t}+4 e^{-0.7 t}, \\
R_{y}(t)= & (0.5 \epsilon-1) e^{-0.5 t}+(1-0.2 \epsilon) e^{-0.2 t} \\
& -e^{-0.5(t-1)}-e^{-t}+e^{-0.4 t}
\end{aligned}
$$

By using the VIM in Case 1, we construct the following iteration formula:

$$
\begin{aligned}
x_{n+1}(t)= & x_{n}(t) \\
& -\int_{0}^{t}\left(x_{n}^{\prime}(s)-x_{n}(s-1) y_{n}(s-1)\right. \\
& \left.+1000 x_{n}(s)-2 y_{n}^{2}(s)-R_{x}(s)\right) d s, \\
y_{n+1}(t)= & y_{n}(t) \\
& -\int_{0}^{t}\left(y_{n}^{\prime}(s)-\frac{1}{\epsilon}\right. \\
& \times\left(x_{n}(s-1)-y_{n}(s-1)-\left(1+x_{n}(s)\right)\right. \\
& \left.\left.\times y_{n}(s)+R_{y}(s)\right)\right) d s .
\end{aligned}
$$

To get iterate sequence, we start with an initial approximation $x_{0}(t)=e^{-0.5 t}+e^{-0.2 t}, y_{0}(t)=-e^{-0.5 t}+e^{-0.2 t}$ and let $\epsilon=10^{-6}$. By means of formulas (29a) and (29b), we have

$$
\begin{gathered}
x_{1}(t)=e^{-0.5 t}+e^{-0.2 t}, \\
y_{1}(t)=-e^{-0.5 t}+e^{-0.2 t} .
\end{gathered}
$$

Figure 1 shows the efficiency of VIM for SPIVPDs.

Example 4. Consider SPIVPD (cf. [2]):

$$
\begin{gathered}
x^{\prime}(t)=2 x(t-1)+y(t-1)-1000 x(t)+y(t)+R_{x}(t), \\
t>0, \\
\epsilon y^{\prime}(t)=x(t-1)-y(t-1)+3 x(t)-y(t)+R_{y}(t), \\
0<\epsilon \ll 1, \\
x(t)=1+10 e^{-(t+1) / 2}+5 e^{-(t+1) / \epsilon}, \quad t \leq 0, \\
y(t)=-1-9 e^{-(t+1) / 2}+4 e^{-(t+1) / \epsilon}, \quad t \leq 0,
\end{gathered}
$$

where

$$
\begin{aligned}
R_{x}(t)= & 10004 e^{-(t+1) / 2}+\left(4996-\frac{5}{\epsilon}\right) e^{-(t+1) / \epsilon} \\
& -11 e^{-t / 2}-14 e^{-t / \epsilon}+1000
\end{aligned}
$$

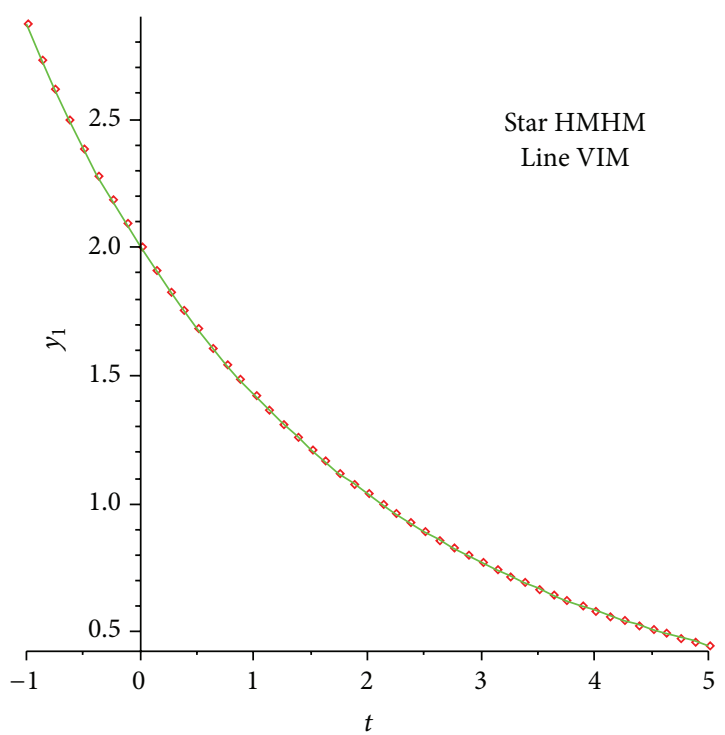

(a)

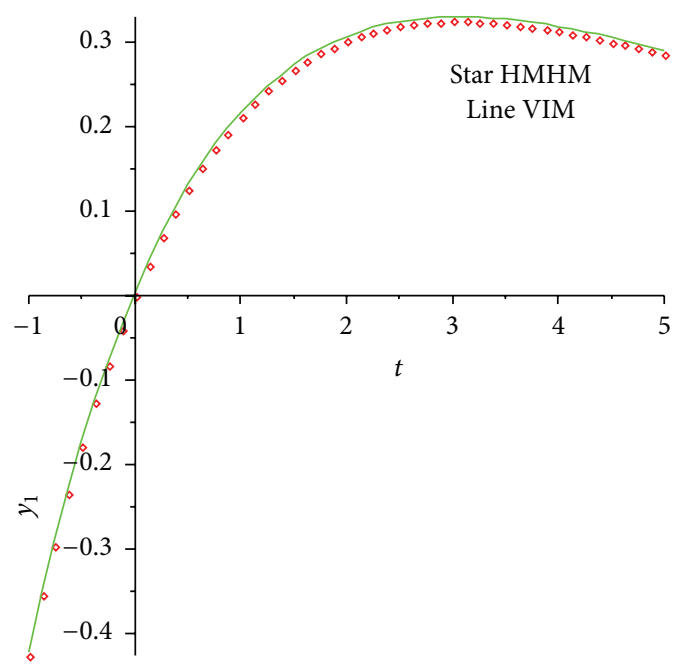

(b)

FIgURE 1: Results for problem 1.

$$
\begin{aligned}
R_{y}(t)= & \left(\frac{9 \epsilon}{2}-39\right) e^{-(t+1) / 2}+15 e^{-(t+1) / \epsilon} \\
& -19 e^{-t / 2}-e^{-t / \epsilon}-6
\end{aligned}
$$

By using the VIM in Case 2, we construct the following iteration formula:

$$
x_{n+1}(t)=x_{n}(t)
$$

$$
\begin{aligned}
-\int_{0}^{t} e^{1000(s-t)}( & x_{n}^{\prime}(s)-2 x_{n}(s-1)-y_{n}(s-1) \\
& \left.+1000 x_{n}(s)-y_{n}(s)-R_{x}(s)\right) d s,
\end{aligned}
$$




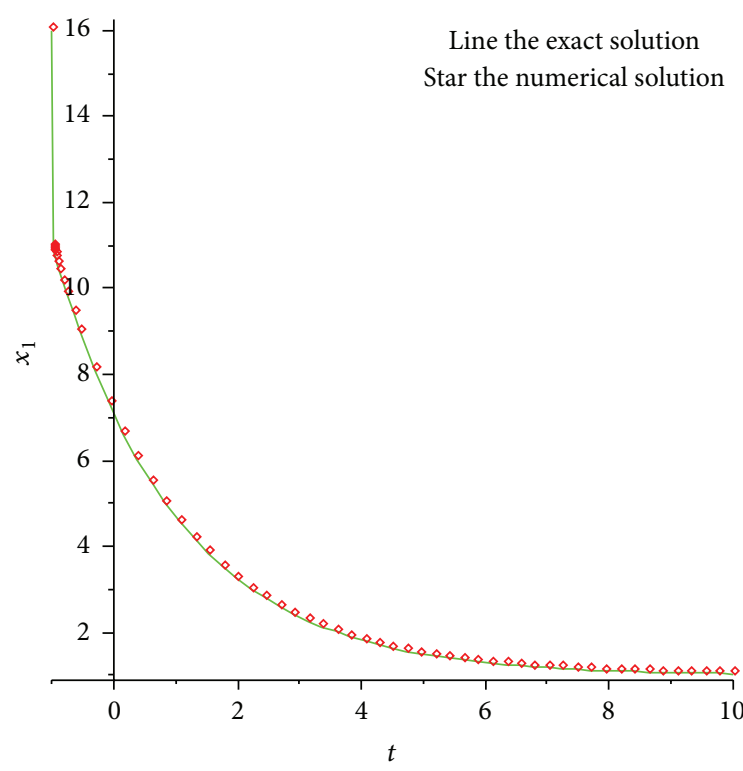

(a)

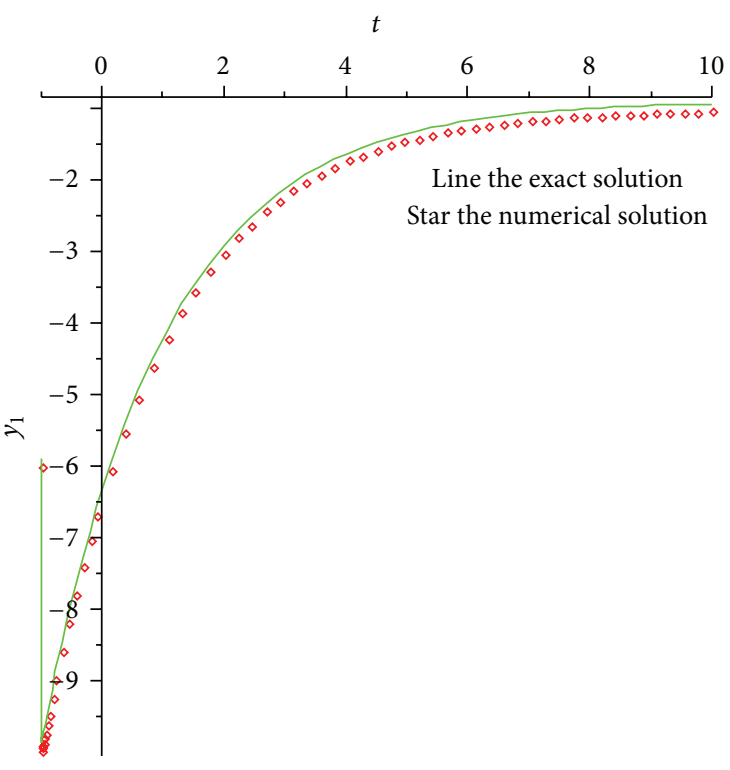

(b)

FIGURE 2: Results for problem 2.

$$
\begin{aligned}
& y_{n+1}(t) \\
& =y_{n}(t)-\int_{0}^{t} e^{(s-t) / \epsilon} \\
& \quad \times\left(y_{n}^{\prime}(s)-\frac{1}{\epsilon}\right. \\
& \quad \times\left(x_{n}(s-1)-y_{n}(s-1)+3 x_{n}(s)\right. \\
& \left.\left.\quad-y_{n}(s)+R_{y}(s)\right)\right) d s .
\end{aligned}
$$

To get iterate sequence, we start with an initial approximation $x_{0}(t)=1+10 e^{-(t+1) / 2}+5 e^{-(t+1) / \epsilon}, y_{0}(t)=-1-$ $9 e^{-(t+1) / 2}+4 e^{-(t+1) / \epsilon}$ and let $\epsilon=10^{-3}$. By means of formulas (33) and (34), we have

$$
\begin{aligned}
& x_{1}(t)=1+10 e^{-(t+1) / 2}+5 e^{-(t+1) / \epsilon} \\
& y_{1}(t)=-1-9 e^{-(t+1) / 2}+4 e^{-(t+1) / \epsilon}
\end{aligned}
$$

Figure 2 shows the efficiency of VIM for SPIVPDs.

\section{Conclusion}

The VIM used in this paper is the variational iteration algorithm I; there are also variational iteration algorithms II and III [29]. In this paper, we apply the VIM to obtain the analytical or approximate analytical solutions of SPIVPDs. The convergence results of VIM for solving SPIVPDs are given. The illustrative examples show the efficiency of the method. When considering the system (16), the choice of correction functionals of Case 1 or Case 2 relies on the practical problems and this choice will result in the difference of the speed of convergence.

\section{Conflict of Interests}

The authors declare that there is no conflict of interests regarding the publication of this paper.

\section{Acknowledgments}

This work is supported by projects NSF of China (11126329, 11271311, and 11201510), Projects Board of Education of Chongqing City (KJ121110), and Key Laboratory for Nonlinear Science and System Structure.

\section{References}

[1] E. M. Dejager and F. R. Jiang, The Theory of Singular Perturbation, Elsevier, Amsterdam, The Netherlands, 1996.

[2] R. E. O'Malley Jr., Singular Perturbation Methods for Ordinary Differential Equations, Springer, New York, NY, USA, 1990.

[3] M. Inokuti, H. Sekine, and T. Mura, "General use of the Lagrange multiplier in nonlinear mathematical physics," in Variational Method in the Mechanics of Solids, S. Nemat-Nasser, Ed., pp. 156-162, Pergamon Press, New York, NY, USA, 1978.

[4] J. H. He, "Some applications of nonlinear fractional differential equation and their approximations," Bulletin of Science, Technology \& Society, vol. 15, no. 12, pp. 86-90, 1999.

[5] J. H. He, "A new approach to nonlinear partial differential equations," Communications in Nonlinear Science and Numerical Simulation, vol. 2, no. 4, pp. 230-235, 1997.

[6] J. H. He, "Approximate solution of nonlinear differential equations with convolution product nonlinearities," Computer Methods in Applied Mechanics and Engineering, vol. 167, no. 1-2, pp. 69-73, 1998.

[7] J. H. He, "A short remark on fractional variational iteration method," Physics Letters A, vol. 375, no. 38, pp. 3362-3364, 2011. 
[8] J. H. He, "Asymptotic methods for solitary solutions and compactons," Abstract and Applied Analysis, vol. 2012, Article ID 916793, 130 pages, 2012.

[9] A. M. Wazwaz, "The variational iteration method for analytic treatment for linear and nonlinear ODEs," Applied Mathematics and Computation, vol. 212, no. 1, pp. 120-134, 2009.

[10] A. M. Wazwaz, "A reliable algorithm for obtaining positive solutions for nonlinear boundary value problems," Computers \& Mathematics with Applications, vol. 41, no. 10-11, pp. 1237-1244, 2001.

[11] G. E. Draganescu and V. Capalnasan, "Nonlinear relaxation phenomena in polycrystalline solids," International Journal of Nonlinear Sciences and Numerical Simulation, vol. 4, no. 3, pp. 219-225, 2003.

[12] G. E. Draganescu, N. Cofan, and D. L. Rujan, "Nonlinear vibrations of a nano sized sensor with fractional damping," Journal of Optoelectronics and Advanced Materials, vol. 7, no. 2, pp. 877-884, 2005.

[13] A. Saadatmandi and M. Dehghan, "Variational iteration method for solving a generalized pantograph equation," Computers \& Mathematics with Applications, vol. 58, no. 11-12, pp. 2190-2196, 2009.

[14] D. K. Salkuyeh, "Convergence of the variational iteration method for solving linear systems of ODEs with constant coefficients," Computers \& Mathematics with Applications, vol. 56, no. 8, pp. 2027-2033, 2008.

[15] J. F. Lu, "Variational iteration method for solving two-point boundary value problems," Journal of Computational and Applied Mathematics, vol. 207, no. 1, pp. 92-95, 2007.

[16] L. Xu, "Variational iteration method for solving integral equations," Computers \& Mathematics with Applications, vol. 54, no. 7-8, pp. 1071-1078, 2007.

[17] M. Rafei, D. D. Ganji, H. Daniali, and H. Pashaei, "The variational iteration method for nonlinear oscillators with discontinuities," Journal of Sound and Vibration, vol. 305, no. 4-5, pp. 614-620, 2007.

[18] M. T. Darvishi, F. Khani, and A. A. Soliman, "The numerical simulation for stiff systems of ordinary differential equations," Computers \& Mathematics with Applications, vol. 54, no. 7-8, pp. 1055-1063, 2007.

[19] M. Tatari and M. Dehghan, "On the convergence of He's variational iteration method," Journal of Computational and Applied Mathematics, vol. 207, no. 1, pp. 121-128, 2007.

[20] M. Mamode, "Variational iterative method and initial-value problems," Applied Mathematics and Computation, vol. 215, no. 1, pp. 276-282, 2009.

[21] R. Saadati, M. Dehghan, S. M. Vaezpour, and M. Saravi, "The convergence of He's variational iteration method for solving integral equations," Computers \& Mathematics with Applications, vol. 58, no. 11-12, pp. 2167-2171, 2009.

[22] Z. H. Yu, "Variational iteration method for solving the multipantograph delay equation," Physics Letters A, vol. 372, no. 43, pp. 6475-6479, 2008.

[23] V. Marinca, N. Herisanu, and C. Bota, "Application of the variational iteration method to some nonlinear one-dimensional oscillations," Meccanica, vol. 43, no. 1, pp. 75-79, 2008.

[24] X. J. Yang and B. Dumitru, "Fractal heat conduction problem solved by local fractional variation iteration method," Thermal Science, vol. 17, no. 2, pp. 625-628, 2013.

[25] G. C. Wu, "Laplace transform overcoming principle drawbacks in application of the variational iteration method to fractional heat equations," Thermal Science, vol. 16, no. 4, pp. 1257-1261, 2012.

[26] Y. X. Zhao and A. G. Xiao, "Variational iteration method for singular perturbation initial value problems," Computer Physics Communications, vol. 181, no. 5, pp. 947-956, 2010.

[27] J. H. He and X. H. Wu, "Variational iteration method: new development and applications," Computers \& Mathematics with Applications, vol. 54, no. 7-8, pp. 881-894, 2007.

[28] J. H. He, "Variational iteration method-some recent results and new interpretations," Journal of Computational and Applied Mathematics, vol. 207, no. 1, pp. 3-17, 2007.

[29] J. H. He, G. C. Wu, and F. Austin, "The variational iteration method which should be followed," Nonlinear Science Letters A, vol. 1, no. 1, pp. 1-30, 2010. 


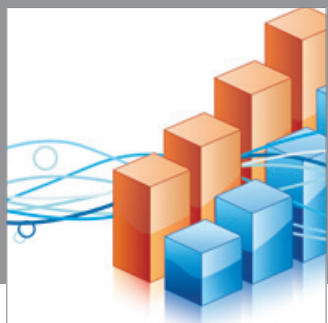

Advances in

Operations Research

mansans

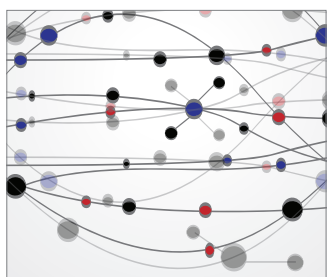

The Scientific World Journal
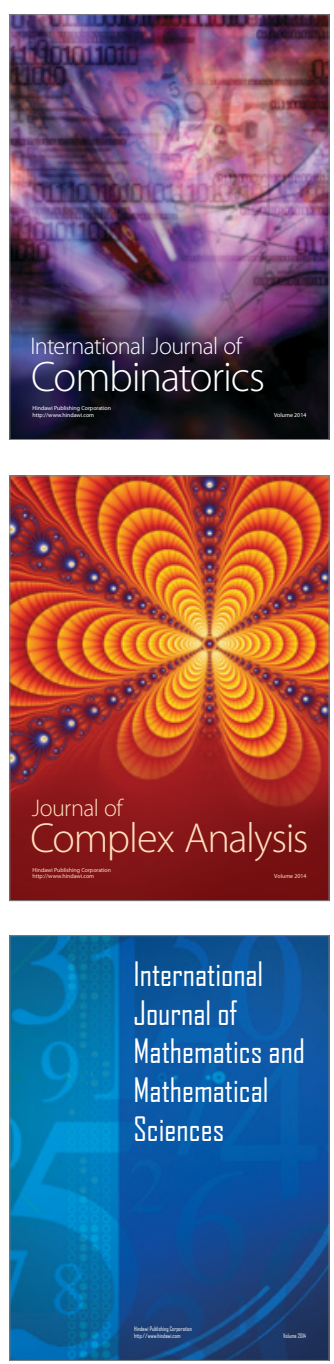
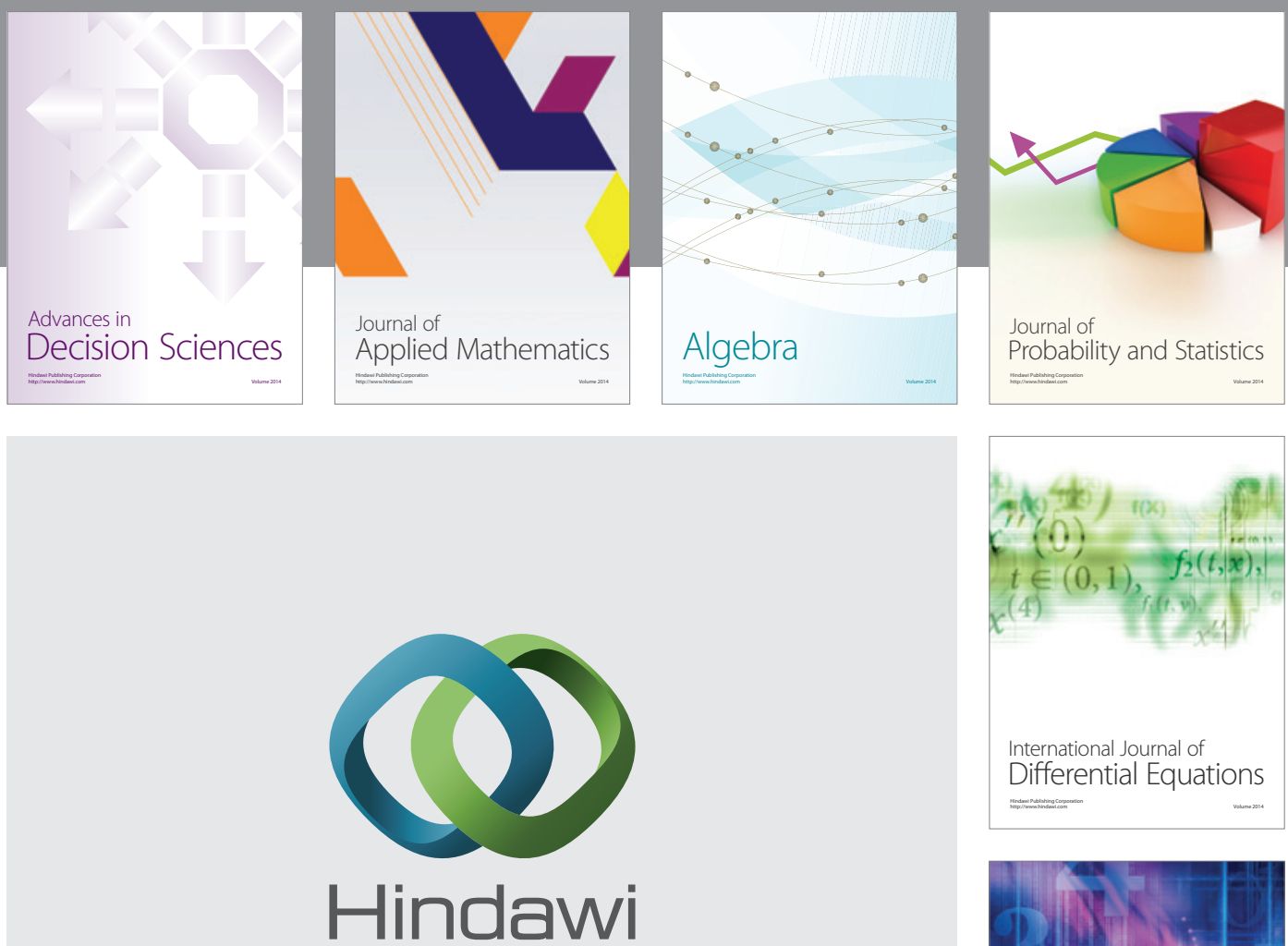

Submit your manuscripts at http://www.hindawi.com
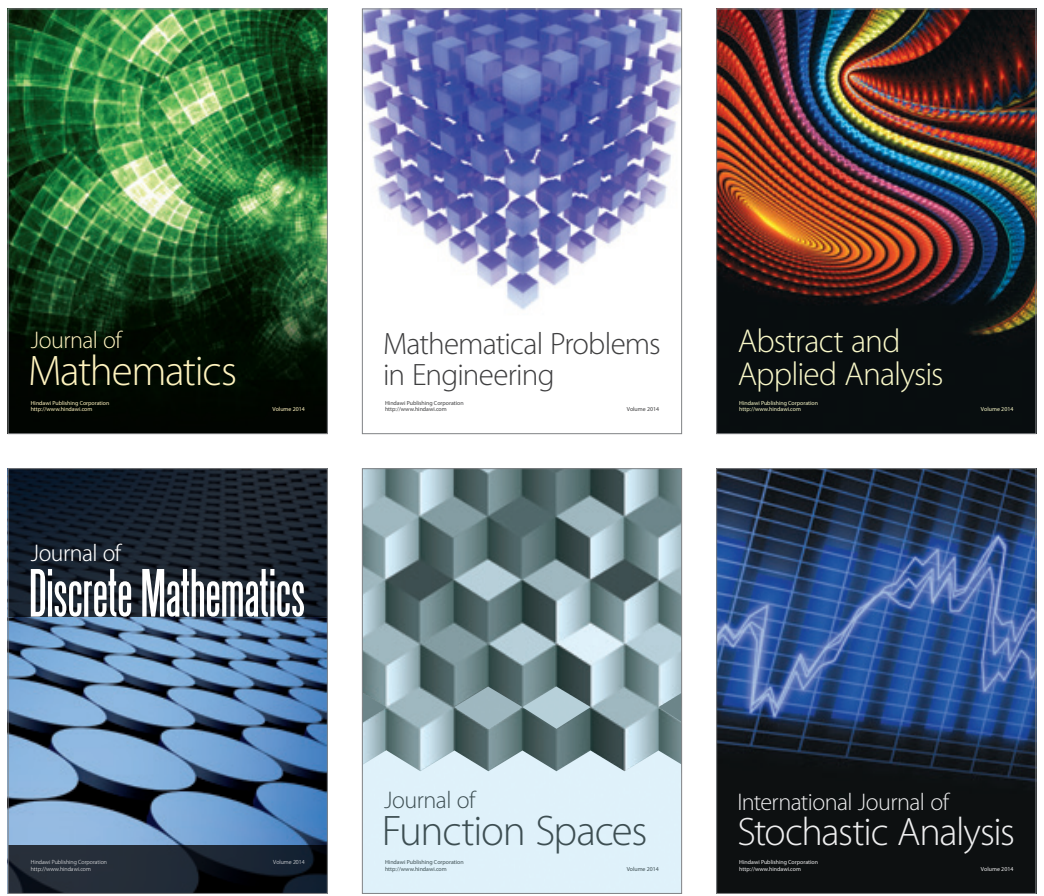

Journal of

Function Spaces

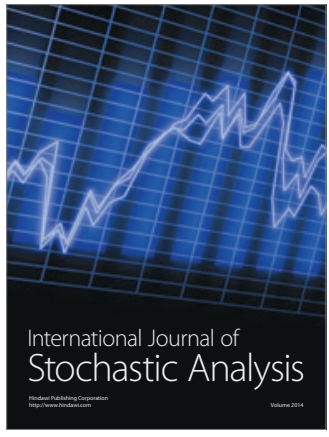

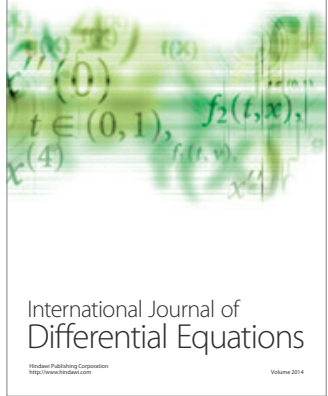
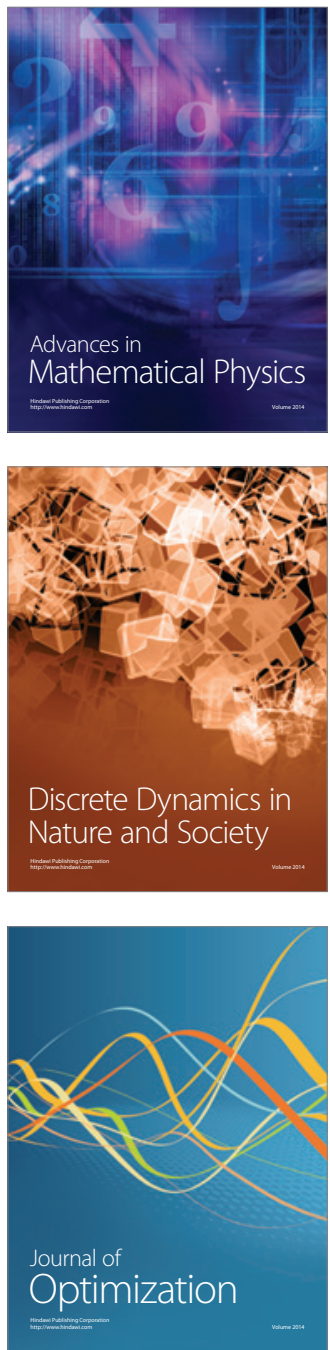